\title{
Prevalence of Benign Paroxysmal Positional Vertigo: Our experiences at a tertiary care hospital of India
}

\author{
Original \\ Article \\ Santosh Kumar Swain ${ }^{1}$, Ishwar Chandra Behera' ${ }^{2}$ Alok Das ${ }^{1}$, Mahesh Chandra Sahu ${ }^{3}$ \\ ${ }^{(1)}$ Department of Otorhinolaryngology, (2)Department of Community Medicine, ${ }^{(3)}$ Medical \\ Research Laboratory - IMS and SUM hospital, Siksha "O” Anusandhan University, K8, Kali \\ nganagar,Bhubaneswar-751003, Odisha, India
}

\begin{abstract}
Introduction: Benign paroxysmal positional vertigo (BPPV) is a common cause of vertigo.BPPV is often overlooked by general physician and treated with anti-vertigo drugs.

Objective: To evaluate the prevalence, incidence,clinical presentation of benign paroxysmal positional vertigo (BPPV). Also assessing the age,gender and site of the lesion, association with other vestibular disorders, progression, and recurrence in these patients.

Materials and methods: A retrospective study of total of 172patients those attended in vertigo clinic for giddiness diagnosed as BPPV. Data analyzed from medical records since last 3 years. Out of total 685 vertigo patients in our Vertigo Clinic, 172were found to suffer from BPPV.The age of the patients ranged from 23 years to 76 years with mean of 41.4 year.

Results: Out of 685 patients with vertigo, 172 were diagnosed as BPPV. Among them, 102 female and 70 male patients. The posterior canal was affected in $81.97 \%$, the lateral in $10.46 \%$, the anterior in $5.23 \%$ and multiple canals in 2.32 $\%$.The treatment maneuver used most was Epley'smaneuver. Recurrence was observed in $10.37 \%$ of the cases. It was idiopathic in $73.83 \%$ of cases.

Conclusion: BPPV is a common peripheral vestibular disorder causing significant morbidity, psychosocial impact and medical costs. Epley's maneuver provides rapid relief of symptoms of BPPV. In addition daily Brandt-Daroff exercises decrease the chances of recurrence rate.
\end{abstract}

Key Words: Benign paroxysmal vertigo, Dix-Hallpike maneuver, Epley’s maneuver, Vertigo.

Received: 22 September 2018, Accepted: 24 December 2018

Corresponding Author: Santosh K. Swain, MS (ORL), DNB(ORL), Department of Otorhinolaryngology, IMS and SUM Hospital, Bhubaneswar, Odisha, India, Tel.: 09556524887, E-mail: santoshvoltaire@yahoo.co.in

ISSN: 2090-0740, November 2018, Vol.19, No. 3

\section{INTRODUCTION}

Benign paroxysmal positional vertigo (BPPV) is characterized by brief periods of vertigo triggered by a changing head position relative to gravity. ${ }^{[1]}$ It is the most common peripheral vestibular disorder, accounting for one third of vestibular diagnosis in the population. ${ }^{[2]}$ Its incidence varies between 11 to 64 cases per 100,000 populations. ${ }^{[3]}$ It is common in the age between 50 and 55 years in idiopathic cases ${ }^{[4]}$ and it is rarely seen in childhood. It is more commonly seen in older ages because of the degeneration of statoconia, arising from demineralization, shown in histopathology studies. ${ }^{[5]}$

\section{PREVALENCE AND INCIDENCE}

The lifetime prevalence of BPPV was $3.2 \%$ in females, $1.6 \%$ in males and $2.4 \%$ overall. The 1 year incidence was calculated as $0.6 \%$. Based on these estimates, 1.1 million adults suffer from BPPV each year in Germany. ${ }^{[6]}$ Furthermore, BPPV is likely to be the most common vestibular disease as every third participant with vestibular vertigo had $\mathrm{BPPV}_{\mathrm{C}^{[7]}}$ Previous studies that aimed to assess the epidemiology of BPPV were subject to various methodological limitations and provided considerably lower incidence estimates. A study from Japan reported an incidence of BPPV of $0.01 \%$ but this can be regarded as an underestimation as only patients presenting during the acute stage to physicians affiliated with the research committee were included. ${ }^{[8]}$ Another study from Olmsted County, Minnesota estimated the incidence of BPPV at $0.06 \%$ on the basis of a population based medical records linkage system. ${ }^{[7]}$ However, patients not seeking medical help were not included and thus this study does not appear to be representative of the general population. ${ }^{[9]}$ Furthermore, both studies relied on a retrospective analysis of medical records which can be a source of inclusion bias. A cross-sectional study that aimed to identify un-recognized BPPV in a group of 100 unselected elderly patients with multiple chronic diseases found a strikingly high point prevalence of $9 \%{ }^{[10]}$ 
BPPV is characterized by paroxysmal positional nystagmus, which may be torsional, vertical, or horizontal, and is characterized by findings such as latency, crescendo and decrescendo, transience, reversibility, and fatigability. ${ }^{[10]}$ Although in most cases of BPPV the posterior canal is affected, BPPV of the horizontal canal, ranging from 5\% to $30 \%{ }^{[12]}$ and more rarely anterior canal involved. ${ }^{[13]}$ The diagnosis of BPPV is based on clinical history and physical examination without any auditory symptoms. The clinical history is described as vertigo spells by changing head position when patient rolls over to one side in bed, as patient gets up,looks up,bends down and it may be associated with or without nausea or vomiting. The main diagnostic test for the posterior canal BPPV is the Dix Hallpike test, which aims to trigger thelabyrinthine symptom or sign such as vertigo, nausea and/or nystagmus. In case of bilateral Anterior canal BPPV, Dix Hallpike test in both sides produces a mixed nystagmus with a weak torsional and intense vertical component in both occasions to those of posterior semicircular canal involvement. ${ }^{[14]} \mathrm{BPPV}$ is often associated with vestibular neuritis, vertebrobasilar insufficiency, chronic otitis media and following ear surgery ${ }^{[11]} \mathrm{BPPV}$ is often misdiagnosed by clinician and sometimes overinvestigated.Sometimes BPPV is associated with tubo-tympanic type of chronic otitis media. BPPV was first described by Barany in the literature in 1921. ${ }^{[15]} \mathrm{He}$ attributed this lesion with otolithic organs. Dix and Hallpike in 1952 first performed provocative positional testing which was named in their honor. ${ }^{[16]}$ The aim of this study was to estimate the prevalence and clinical profile of BPPV at a tertiary care center in eastern India. There is no such study done in this part of India.

\section{MATERIALS AND METHOD}

Our study was carried out at a tertiary care teaching hospital of eastern India from December 2015 to August 2018. Data from medical reports of BPPV patients were analyzed during the period of past 3 years. The Institutional Ethics Committee(IEC) of our institution approved the study. Detail patients profile like age, sex, etiologies and clinical presentations were analyzed. Clinical data were collected from the files of consecutive patients seen within the last 3 years, in whom BPPV was diagnosed by neurootologist or Otorhinolaryngologists.Patients of BPPV were included in our study by considering symptoms with episodes of rotatory dizziness with changes in the position of the head, when lying on one or both sides. Other clinical symptoms were nausea with or without vomiting, and other types of dizziness; positional or positioningnystagmus could also be present.Patients with features of central nervous system involvement were excluded from our study. Patients with hearing loss detected in pure tone audiometry were excluded from the study. Detail clinical and neuro-otological examinations were carried out among all patients complaining vertigo at our Vertigo Clinic. Nystagmus noted if any and cerebellar functions like asynergia,asymmetria and dysdiadokinesisare elicited to rule out any cerebellar etiology for vertigo.Dix-Hallpike maneuver is the gold standard clinical test done for BPPV. This test was done among all patients complaining vertigo. If this maneuversshows nystagmus associated with vertigo, it confirms the diagnosis.If it does not evoke any kind of nystagmus, it is against diagnosis of BPPV.The BPPV of posterior semicircular canal typically shows geotropic rotatory nystagmus and vertigo. The fast component of the nystagmus is always towards lowermost ear(affected ear) whereas purely horizontal nystagmus represents horizontal semicircular canal involvement. ${ }^{[11]}$ The treatment options of BPPV without nystagmus may be done on the basis of the typical history of BPPV and signs found upon physical examination, with vertigo. One should treat the side on which the signs were triggered by means of the Epley's maneuver and Sémont's maneuvers and the Brandt-Daroff exercises, or even, by the modified freeing maneuver for the posterior semicircular canal.

\section{Dix-Hallpike test}

We had done Dix-Hallpike test(Fig.1) in all cases of vertigo for confirming the BPPV among those were coming with complaints of vertigo. If patient is using spectacles, it should be removed before starting maneuver. The posterior canal and horizontal canal were clinically differentiated by observing the nystagmus during the maneuver. Posterior canal: In Dix-Hallpike maneuver shows torsional nystagmus, in which the upper pole of the eye rotates toward the affected side where the patient is brought from the upright to supine position with the head turned 450 to the affected side. The nystagmus contains an additional vertical (upward) component. Horizontal canal: Supine roll test shows geotropic positional nystagmus: rightward horizontal nystagmus is induced by the right-ear-down head position whereas leftward horizontal nystagmus is induced by the left-ear-down head position in the supine position of the patient. The nystagmus consists of mainly horizontal and minor torsional components .In anterior canal BPPV, the nystagmus is observed when the patient is moved into the Dix-Hall pike position, with the direction of the fast phase being down beating and torsional.

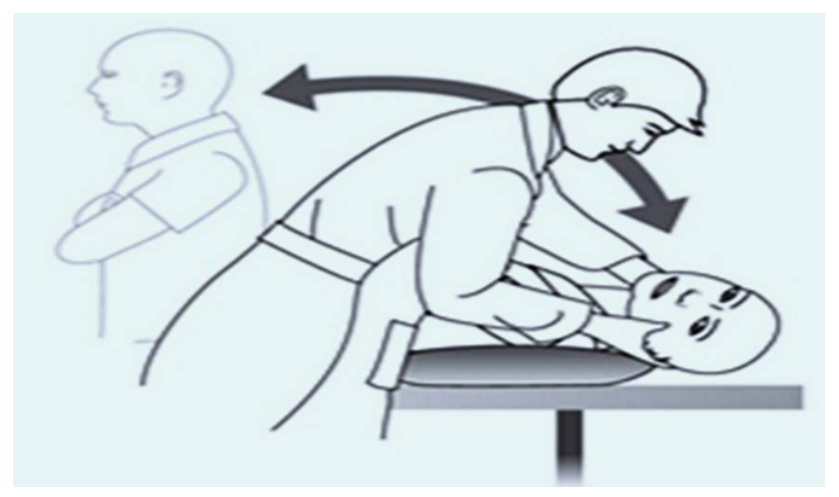

Fig 1: Picture showing Dix-Hallpike test 


\section{Treatment}

Medical treatment with vestibular sedatives is not much useful in BPPV.The treatment of choice in BPPV is Epley's maneuver(Fig.2).This maneuver has five steps. Position.1:Patient sits on the examination couch and head turned towards affected ear by 450 and then patient rapidly brought downwards in head hanging position. Then nystagmus appears after 20-30 seconds which is called as latent period.Head will stay in this position till the nystagmus disappears.Position.2:Head is turned to unaffected side and keep for 2 minutesin this position. Position.3:The whole body and head are turned away from the affected ear to a lateral recumbent position and wait for 2 minutes.Position.4:Patient is come to the sitting position with head turned away from the affected side by 450 and wait for 2 minutes.Position.5:In sitting position,patient brings his face forwards with chin 200 downwards and wait for 2 minutes. After Epley's maneuver, all patients were advised for sleeping with elevated head (posterior/anterior canal), not lying on the side of disease, sleeping all night with the healthy ear down (horizontal canal) and avoiding cervical extension or rotation for maximum 8hours.
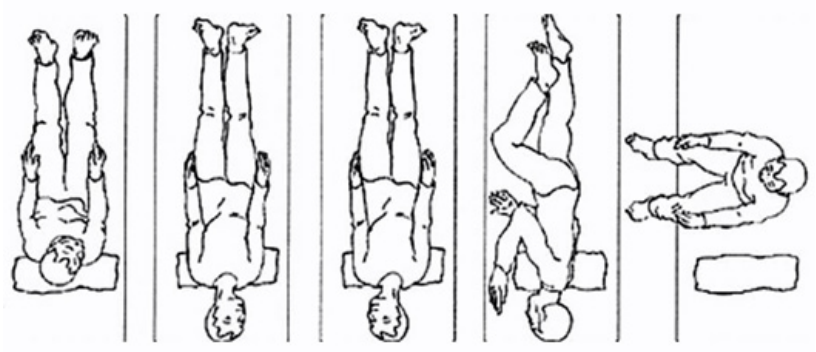

Fig 2: Picture showing steps of Epley's maneuver

\section{RESULTS}

There were 172 patients of BPPV included in our study out of 685 vertigo patients attended the Vertigo Clinic of our center. Out of 172 BPPV patients, 102 females and 70 males with an age range of 23-76 years (mean age 41.4 years) were found. Duration of clinical manifestations before diagnosis ranged from 2 to 20 days. Among them, 104 patients $(60.46 \%)$ presented with first episode of BPPV whereas 68 patients $(39.53 \%)$ presented with recurrent attack of BPPV Table (1).The most common etiology is idiopathic in 127 cases $(73.83 \%)$, followed by head trauma in 15 cases $(8.72 \%)$, migraine in 15 cases $(8.72 \%)$, Ménière's disease in 8 cases (4.65\%) and others in 7 cases (4\%) (Demyelinating disease, labyrinthitis, cerebellopontine angle lesions) Table (2). Female out numbered males in the idiopathic group in our study.The most common clinical presentation is rotational vertigo, which is commonly experienced during turning over in bed Table (3). The posterior semicircular canal was affected more commonly in 141 cases $(81.97 \%)$ than the lateral in 18 cases $(10.46 \%)$ and anterior in 9 cases $(5.23 \%)$ canals, multi-canal in 4 cases $(2.32 \%)$ (posterior/anterior). Unilateral canal involvement in 164 cases $(95.34 \%)$ was more frequent than bilateral canal involvement in 8 cases $(4.65 \%)$. Left labyrinth involvement was more frequent affected relatively to the right one (48.5\% vs $46.5 \%)$. The therapeutic maneuver used was Epley's maneuver in 121 cases(70.4\%), followed by Brandt Daroff exercises in 42 cases $(24.4 \%)$, Semont in 2 cases $(1.2 \%)$ and Barbecue in 7 cases $(4 \%)$ Table (4). The number of therapeutic maneuvers done at diagnosis was one in 142 cases $(82.5 \%)$, two in 21 cases $(12.2 \%)$, three in 2 cases $(1$. $2 \%$ ) and 7 patients that refused or were too symptomatic at the moment of diagnosis(4\%). About 62 cases $(36 \%)$ of patients repeated maneuvers (due to failures) at reevaluation. Recurrence was seen in 23 cases $(10.37 \%)$ and 38 cases $(22 \%)$ had previous episodes, similar or but less intense .Reverse Epley maneuver, the patients do the same sequence of positional changes after Dix-Hallpike on the side of healthy ear. More treatment sessions are required for multiple canal BPPV.

Table 1: Descriptive table for BPPV Patients

\begin{tabular}{lcc}
\hline Age & Age range:23-76 years & Mean:41.4 years \\
\hline Sex & Female:102(59.30\%) & Male:70(40.69) \\
Presentation & First presentation: & Recurrent episodes: \\
& $104(60.46 \%)$ & $68(39.53 \%)$ \\
\hline
\end{tabular}

Table 2: Etiological profile of BPPV

\begin{tabular}{lcc}
\hline Causes & Number of patients(n) & Percentage (\%) \\
\hline Idiopathic & 127 & $73.83 \%$ \\
Head trauma & 15 & $8.72 \%$ \\
Migraine & 15 & $8.72 \%$ \\
Meniere's disease & 8 & $4.65 \%$ \\
Others & 7 & $4 \%$ \\
\hline
\end{tabular}

Table 3: Clinical presentations of BPPV patients

\begin{tabular}{lc}
\hline Clinical features & Percentage \\
\hline Rotational Vertigo & $81 \%$ \\
Nausea & $35 \%$ \\
Vomiting & $13 \%$ \\
Imbalance & $38 \%$ \\
Fear of falling & $31 \%$ \\
Turning over in bed causing vertigo & $88 \%$ \\
Oscillopsia & $2 \%$ \\
Falls due to head movement & $1 \%$ \\
Precipitating head movement & $81 \%$ \\
\hline
\end{tabular}


Table 4: Different therapeutic maneuvers used in BPPV patients

\begin{tabular}{ll}
\hline Name of maneuvers & Number of patients \\
\hline Epley's maneuver & $121(70.4 \%)$ \\
Brandt-Daroff & $42(24.4 \%)$ \\
Barbecue & $7(4 \%)$ \\
Semont's & $2(1.2 \%)$ \\
& Total $=172(100 \%)$ \\
\hline
\end{tabular}

\section{DISCUSSION}

Among inner ear disorders causing vertigo, BPPV is by far the most common. Although the most common cause of BPPV is idiopathic, head trauma, Ménière's disease and ear surgery have been accepted as other causes. This clinical entity is usually easily diagnosed and readily treatable with a simple office bases procedure. Diabetes mellitus, hypertension, hyperlipidemia and vertebrobasilar ischemia are thought to be predisposing factor for BPPV. ${ }^{[17]}$ This may be due to labyrinthine ischemia which causes detachment of otoconia from the otolith membrane. However there are no such studies for explaining the association between BPPV and different systemic diseases. Migraine has been found to be associated with BPPV. There is increased incidence of migraine in patients with BPPV and higher chance of recurrence of BPPV after successful repositioning therapy in patients with migraine. It has been thought that spasm of the inner ear vessels may be a possible etiology as it is well documented in migraine ${ }^{[18]}$ The vertigo in BPPV is typically intense in nature and is aggravated by head movements in horizontal or sagittal plane that is in the plane of affected semicircular canal. In BPPV, there is often an earlier history of similar symptoms. In a study it was revealed that a repeated CRP in patients with post-CRP dizziness increased the rate of recovery from dizziness but had no influence on BBPV recurrence. ${ }^{[19]}$ In another study provided the classification, diagnostic criteria, the examinations for diagnosis, and specific and non-specific treatments of the posterior- and lateral-canal- type of BPPV in accordance with the Japanese practical guidelines on BPPV published by the Japan Society for Equilibrium Research. ${ }^{[20]}$ Patient of BPPV have no auditory or neurologic symptoms. There are multiple etiology of vertigo which may be confused with BPPV and may be divided in otological causes: Ménière's disease, labyrinthitis with hearing loss(HL), vestibular neuritis, superior canaldehiscence syndrome, posttraumatic vertigo; neurological causes:migrainous dizziness, vertebrobasilar insufficiency, demyelinating disorders and CNS lesions with HL; and others: anxiety disorder or panic disorder, cervicogenic vertigo, drug side effects, postural hypotension. ${ }^{[21]}$ The posterior canal BPPV is more commonly seen than the lateral and anterior canals. This may be due to the spatial position of the posterior semicircular canal which is more favorable to the migration of statoconia from the utricle. ${ }^{[22]}$ Posterior canal BPPV is characterized by rotatory and upbeat vertical positional nystagmus (counterclockwise in right labyrinth lesions and clockwise in left labyrinthlesions).This is the most common type of BPPV accounting for up to $90 \%$ of the patients. ${ }^{[23]}$ The incidence of posterior canal BPPV in our study is $81.97 \%$. Anterior canal BPPV is a rare clinical entity and its incidence has been reported to be within range from $1 \%$ to $15 \%$. Anterior canal BPPV is characterized by rotatory and downbeat vertical positional nystagmus (counterclockwise in right labyrinth lesions and clockwise in left labyrinth lesions). Exclusively counterclockwise or clockwise rotatory positional nystagmus indicates involvement of the vertical canal; even not specify which vertical canal is involved. In vertical canal involvement, canalolithiasis is defined by nystagmus lasting up to $1 \mathrm{~min}$, and cupulolithiasis is evidenced by nystagmus persisting more than 1 minute. ${ }^{[24]}$ Lateral or horizontal semicircular canal BPPV is characterized by horizontal positional or positioning nystagmus. Horizontal positional nystagmus is geotropic when patient tilting the head to the right, causes right horizontal nystagmus and tilting the head to theleft causes left horizontal nystagmus. It is ageotropic in nature when tilting the head to the right causes left horizontal nystagmus and tilting the head to the left causes right horizontal nystagmus. BPPV originating from stimulation of the lateral semicircular canal is the second most common type of BPPV, accounting for approximately 5\% to $15 \%$ of the patients. Its frequency has been sometimes reported up to30 \%. ${ }^{[13]}$ Whereas in our study, it is $10.46 \%$. The delay in diagnosis of BPPV is due to lack of clinician's awareness of this lesion.The delayed diagnosis is sometimes attributed to the atypical presentation of BPPV.A study reported that $80 \%$ of patients experience a rotatory vertigo and $47 \%$ presents with floating sensation. ${ }^{[25]} \mathrm{BPPV}$ accounts for $8 \%$ of the patients suffering from moderate to severe vertigo. ${ }^{[19]}$ It may be seen among children but increases with increase in age. The lifetime prevalence is around $2 \%$. It is documented that one year prevalence is $0.5 \%$ in $18-39$ years of age and 3.4\% among people over 60 years of age. ${ }^{[26]}$ In case of BPPV, without any treatment, the symptoms gradually disappear over a period of two to four weeks.However, if the symptom persists, a repositioning maneuver is advocated. Drugs like vestibular suppressant are not effective as a definitive treatment for BPPV. A technique for a repositioning of the particles from posterior semicircular canal was designed by Semont(1988) and Epley(1992). ${ }^{[27]}$ Both techniques are based on the same assumption of semicircular canal and are positioned into the utricle by utilizing the gravity force. The Epley'smaneuver is commonly used technique for posterior semicircular canal BPPV. Particle repositioning maneuvers are the recom $\neg$ mended for treatment of BPPV. This is effective for most of the posterior canal BPPV. Recurrences may need repeated therapy. ${ }^{[28]}$ BPPV is a benign vestibular disease where surgery should only be reserved for the most intractable or multiply recurrent cases. Before considering surgery, the brain should be imaged to rule out any central organic lesions that might mimic BPPV. Singular neurectomy, or sectioning the posterior ampullary nerve, which sends impulses from the 
posterior semicircular canal to the brain, was initiated and popularized by Gacek in the 1970s. ${ }^{[29]}$ There was a risk of sensorineural hearing loss, but the surgery has been found to be technically demanding. Now days it has largely been replaced by the simpler technique like posterior semicircular canal occlusion.

\section{CONCLUSION}

BPPV is a commonly found peripheral vestibular disorder leading to significant morbidity, psychosocial impact,medical costs and burden to family.BPPV is more prevalent over the age of 45 years and more among female patients.Most cases are with idiopathic etiology.Most of the cases present dizziness and positioning nystagmus due to right posterior canal canalolithiasis. The vestibular disease like Ménière's is often associated with BPPV. Although Canalolith repositioning maneuver is safe and effective treatment, still there is chance of recurrence.

\section{REFERENCES}

1. Helminski JO, Zee DS, Janssen I, Hain TC. Effectiveness of particle repositioning maneuvers in the treatment of benign paroxysmal positional vertigo: a systematic review. PhysTher. 2010; 90:663-678

2. Von Brevern M, Radtke A, Lezius F, et al. Epidemiology of benign paroxysmal positional vertigo: a population based study. J Neurol Neurosurg Psychiatry. 2007;78:710-714

3. Maia RA, Diniz FL, Carlesse A. Manobras de reposicionamento na vertigem paroxística posicional benigna. Rev Bras Otorrinolaringol. 2001;67(5):612-616.

4. Hilton M, Pinder D. The Epley manoeuvre for benign paroxysmal positional vertigo - a systematic review. Clin Otolaryngol Allied Sci.2002; 27(6):440-445.

5. Walther LE, Westhofen M. Presbyvertigo-aging of otoconia and ves $\neg$ tibular sensory cells. J Vestib Res. 2007; 17(2-3):89-92.

6. Pérez-Vázquez P, Franco-Gutiérrez V. Treatment of benign paroxysmal positional vertigo. A clinical review. Journal of Otology. 2017 Jan 1;12(4):16573.

7. Von Brevern M, Radtke A, Lezius F, Feldmann M, Ziese T, Lempert T, Neuhauser H. Epidemiology of benign paroxysmal positional vertigo: a population based study. Journal of Neurology, Neurosurgery \& Psychiatry. 2007;1;78(7):710-715.
8. Mizukoshi K, Watanabe Y, Shojaku H. et al Epidemiological studies on benign paroxysmal positional vertigo in Japan. Acta Otolaryngol. 1988; 447(Suppl):67-72.

9. Froehling D A, Silverstein M D, Mohr D N. et al Benign positional vertigo: incidence and prognosis in a population-based study in Olmsted County. Minnesota.Mayo Clin Proc. 1991;66: 596-601.

10. Oghalai J S, Manolidis S, Barth J L. et al Unrecognized benign paroxysmal positional vertigo in elderly patients. Otolaryngol Head Neck Surg. 2001; 22:630-634.

11. Korres SG, Balatsouras DG. Diagnostic, pathophysiologic, andtherapeutic aspects of benign paroxysmal positional vertigo.OtolaryngolHead Neck Surg. 2004;131:438-444.

12. De Stefano A, Dispenza F, Suarez H, PerezFernandez N, Manrique-Huarte R, Ban JH, et al. A multicenter observational study on the role of co-morbidities in the recurrent episodes of benign paroxysmal positional vertigo. Auris Nasus Larynx. 2014; 41:31-36.

13. Uno A, Moriwaki K, Kato T, et al. Clinical features of benign paroxysmal positional vertigo. Nippon Jibiinkoka Gakkai Kaiho. 2001;104 :9-16.

14. Balatsouras DG, koukoutsis G, Ganelis P, Korres GS, Kaberos A. Diagnosis of single or multiple canal benign paroxysmal positional vertigo according to the type of nystagmus. Int $\mathrm{J}$ Otolaryngol.2011. Article ID 483965, 13 pp.

15. Oliveira AKS, Suzuki FA, Boari L. Is it important to repeat the positioning maneuver after the treatment for benign paroxysmal positional vertigo? Braz J Otorhinolaryngol. 2015; 81(2):197-201.

16. Patangay KK, Ansari R. Benign paroxysmal positional vertigo.Our Experience. India $\mathrm{J}$ Otolaryngol Head Neck Surg.2016:68(1):39-41.

17. Elsanadiky HH, Nour YA. Diagnosis and management of posteriorsemicircular canal benign paroxysmal positional vertigo: A practical approach.Egyptian Journal of Ear, Nose, Throat and Allied Sciences. 2015; 16:161-166.

18. Ishiyama A, Jacobson KM, Baloh RW. Migraine and benign positional vertigo. Ann Otol Rhinol Laryngol. 2000;109:377-380. 
19. Tirelli G, Nicastro L, Gatto A, Tofanelli M. Repeated canalith repositioning procedure in BPPV: Effects on recurrence and dizziness prevention. American Journal of Otolaryngology. 2017; 38(1):38-43.

20. Imai $\mathrm{T}$, Takeda $\mathrm{N}$, Ikezono $\mathrm{T}$, Shigeno $\mathrm{K}$, Asai $\mathrm{M}$, Watanabe Y, Suzuki M. Classification, diagnostic criteria and management of benign paroxysmal positional vertigo. Auris Nasus Larynx. 2016 ; doi. org/10.1016/j.anl.2016.03.013.

21. Bhattacharyya N, Baugh RF, Orvidas L, Barrs D, Bronston LJ, CassSet., et al. Clinical practice guideline: benign paroxysmal posi-tional vertigo. Otolaryngol Head Neck Surg. 2008; 139:47-81.

22. Caldas MA, Gananc,a CF, Gananc,a FF, Gananc, a MM, Caovilla HH. Clinical features of benign paroxysmal positional vertigo. Braz $\mathrm{J}$ Otorhinolaryngol. 2009; 75:502-506.

23. Balatsouras DG, koukoutsis G, Ganelis P, Korres GS, KaberosA. Diagnosis of single or multiple-canal benign paroxysmal positional vertigo according to the type of nystagmus. Int $\mathrm{J}$ Otolaryngol. 201;14:2011.
24. Hornibook J. Benign paroxysmal positional vertigo (BPPV): history, pathophysiology office treatment and future directions.Int J Otolaryngol. 2011. Article ID 835671, 13 pp.

25. Kentala E, Pyykko I. Vertigo in patients with benign paroxysmal positional vertigo. Acta Otolaryngol Suppl. 2000;543:20-22.

26. Silva C, Amorim AM, Paiva A. Benign Paroxysmal Positional Vertigo-A Review of 101 Cases. Acta Otorrinolaringol Esp. 2015;66(4):205-209

27. Bergeniu J, QingZ,MaoliD. Benign paroxysmal positional vertigo. Journal of Otology. 2014; 9(1):1-6.

28. Steenerson RL, Cronin GW, Marbach PM. Effectiveness of treatment techniques in 923 cases of benign paroxysmal positional vertigo. Laryngoscope. 2005;115:226-231.

29. Parnes LS, Agrawal SK, Atlas J. Diagnosis and management of benign paroxysmal positional vertigo (BPPV). JAMC. 2003; 169 (7):681-693. 